\title{
A PROSPECTIVE, RANDOMIZED TRIAL TO COMPARE TACROLIMUS AND PREDNISONE WITH AND WITHOUT MYCOPHENOLATE MOFETIL IN PATIENTS UNDERGOING RENAL TRANSPLANTATION: FIRST REPORT
}

\author{
RON SHAPIRO,* MARK L. JORDAN, VELMA P. SCANTLEBURY, CARLOS VIVAS, \\ H. ALBIN GRITSCH, F. ADRIAN CASAVILLA, JERRY MCCAULEY, JAMES R. JOHNSTON, \\ PARMJEET RANDHAWA, WILLIAM IRISH, THOMAS R. HAKALA, JOHN J. FUNG AND \\ THOMAS E. STARZL
}

From the Thomas E. Starzl Transplantation Institute, Division of Urologic Surgery, Department of Medicine and Division of Transplant Pathology, University of Pittsburgh, Pittsburgh, Pennsylvania

\section{ABSTRACT}

Purpose: Between September 20, 1995 and September 20, 1996, 120 patients were entered into a prospective, randomized trial comparing tacrolimus and prednisone with (61) and without (59) $2 \mathrm{gm}$. mycophenolate mofetil daily to determine whether mycophenolate mofetil was associated with a lower incidence of rejection.

Materials and Methods: Mean recipient age plus or minus standard deviation was $50.8 \pm 14.1$ years (range 18.8 to 84.1 ). Mean donor age was $34.3 \pm 21.7$ years (range 0.01 to 76 ). Of the donors $18(15 \%)$ were older than 60 years. Mean cold ischemia time was $30.9 \pm 8.4$ hours (range 14.2 to 49). Median followup was $8.6 \pm 0.5$ months.

Results: The 6-month actuarial patient survival was $95 \%, 92 \%$ in the double therapy group and $98 \%$ in the triple therapy group (not significant). The 6-month actuarial graft survival was $88 \%$, $84 \%$ in the double therapy group and $92 \%$ in the triple therapy group (not significant). The overall incidence of rejection and steroid resistant rejection was 34.2 and $4.2 \%$, respectively. There was a strong trend toward less rejection in the mycophenolate mofetil group than in the double therapy group ( 26.2 versus $42.4 \%$ ). Crossover was common, and was $42.6 \%$ from triple to double therapy and $18.6 \%$ from double to triple therapy. The reasons for discontinuation of mycophenolate mofetil were gastrointestinal toxicity, primarily diarrhea, or less commonly hematological toxicity, primarily neutropenia or thrombocytopenia. Gastrointestinal toxicity was ameliorated by separating the doses of tacrolimus and mycophenolate mofetil by 2 to 4 hours, and reducing the dose to $1 \mathrm{gm}$. daily.

Conclusions: Mycophenolate mofetil appears to be a useful third agent with tacrolimus in patients undergoing renal transplantation, and is associated with a reduction in the rate of rejection and a low incidence of steroid resistant rejection. There is a high incidence of gastrointestinal toxicity associated with the $2 \mathrm{gm}$. daily dose but this complication is relatively straightforward to manage.

\section{KEY WORDS: prednisone, kidney transplantation, drug therapy}

Although renal transplantation under tacrolimus based immunosuppression has been associated with excellent short and medium-term outcomes, ${ }^{1-7}$ some of the earlier studies, particularly those not using induction antilymphocyte preparations, have described relatively high rates of early acute rejection ranging from 50 to $60 \% .^{8-12}$ While the majority of these rejection episodes have been steroid responsive, patients with steroid resistant rejection have had markedly inferior outcomes with a 3-year actuarial graft survival of $53 \% .^{2}$ An early study which looked at azathioprine as a third agent demonstrated a modest reduction in the incidence of rejection and steroid resistant rejection from 55 to $45 \%$ and 14 to $8 \%$, respectively. ${ }^{1.2}$ Unfortunately, overall graft survival was not improved, and was actually worse at 3 years in patients not receiving azathioprine ( 76 versus $84 \%$ of patients randomized to double therapy). ${ }^{2.3}$

A new azathioprine substitute, mycophenolate mofetil, was approved by the Food and Drug Administration in June 1995,

Accepted for publication July 28, 1998.

* Requests for reprints: 4th Floor Falk, 3601 Fifth Ave., Pittsburgh, Pennsylvania 15213. having been evaluated in 3 large prospective, randomized trials with cyclosporine based immunosuppression. ${ }^{13-16}$ In these trials the incidence of early acute rejection was reduced significantly from 41 to 17 to $20 \%$, although the 1-year patient and graft survival rates were unaffected. In an attempt to determine whether mycophenolate mofetil would offer similar benefits with tacrolimus based therapy, we began a prospective, randomized trial of tacrolimus and prednisone with and without mycophenolate mofetil in adult renal transplant recipients. Azathioprine was not used in the control group because of the inferior outcomes noted in an earlier randomized trial. ${ }^{2.3}$ In this initial report we evaluate the first year of the trial with a relatively short followup, since as yet there are no clinical reports on the combination of tacrolimus and mycophenolate mofetil in a randomized trial.

\section{PATIENTS AND METHODS}

Recipient and donor characteristics. Between September 20, 1995 and September 20,1996, 120 adult patients undergoing first or second cadaveric kidney transplantation only 
were entered into a prospective, randomized open label trial of tacrolimus and prednisone only (59) versus tacrolimus and prednisone with mycophenolate mofetil (61, table 1$)$. Induction antilymphocyte therapy was not used. Pediatric patients, those undergoing the third or greater transplantation, those receiving concomitant multiple organs including bone marrow augmentation, those receiving kidneys from living donors and those refusing to consent were excluded from the trial. Mean recipient age was $50.8 \pm 14.1$ years (range 18.8 to 84.1). Of the patients $17(14.2 \%)$ were undergoing the second transplantation and $11(9.2 \%)$ had previously received a liver (10) or heart (1) allograft. Four patients (3.3\%) were sensitized with a panel reactive antibody level greater than $40 \%$, and $38(31.7 \%)$ were older than 60 years at the time of transplantation.

Mean donor age was $34.3=21.7$ years (range 0.01 to 76 ) Of the transplants $16(13 \%)$ were pediatric en bloc kidneys from donors 3 years old or younger. Of the donors $18(15 \%)$ were older than 60 years. Mean cold ischemia time was $30.9 \pm 8.4$ hours (range 14.2 to 49 ). Cold storage preservation was used. Mean number of human leukocyte antigen matches and mismatches was $2.6 \pm 1.2$ and $3.1 \pm 1.3$, respectively. There were 7,0 antigen mismatched cases $(5.8 \%)$.

Immunosuppression. A dose of $0.15 \mathrm{mg} . / \mathrm{kg}$. tacrolimus was given orally to all patients preoperatively. In the recovery room or intensive care unit 0.025 to $0.05 \mathrm{mg} . / \mathrm{kg} . / 24$ hours intravenous tacrolimus was given as a continuous infusion. When patients were able to tolerate oral medications 0.15 $\mathrm{mg} / \mathrm{kg}$. tacrolimus orally twice a day was begun and intravenous tacrolimus was tapered off in 0 to 48 hours. Further dosage adjustments were guided by levels. Target tacrolimus levels by whole blood IMx, were 20 to $25 \mathrm{ng} . / \mathrm{ml}$., for the first 2 weeks, 15 to 20 at 1 month, 10 to 15 at 3 months and 5 to 12 chronically.

Steroids. All patients received intraoperatively $1,000 \mathrm{mg}$. methylprednisolone intravenously and a short steroid taper from 200 to 20 or $30 \mathrm{mg}$. daily for 6 days. Further tapering was individualized but in the ideal case the prednisone dose was decreased to $15 \mathrm{mg}$. daily by 3 to 4 weeks and then by 2.5 mg. decrements to $10 \mathrm{mg}$. daily by 2 to 3 months after transplantation. Thereafter the prednisone dose was decreased by $2.5 \mathrm{mg}$. every 4 to 6 weeks until it was completely discontinued, if possible.

Mycophenolate mofetil. Patients randomized to receive mycophenolate mofetil were given $1,000 \mathrm{mg}$. orally preoperatively and started on $1,000 \mathrm{mg}$. orally twice a day beginning on postoperative day 1 . After the first few months of the study tacrolimus and mycophenolate mofetil dosages were separated by 3 to 4 hours, which significantly improved the tolerability of the combination of the 2 agents. In the case of adverse events, for example diarrhea, gastritis, leukopenia or thrombocytopenia, the mycophenolate mofetil dose was generally decreased to $500 \mathrm{mg}$. orally twice a day. If side effects persisted, mycophenolate mofetil was discontinued.

Rejection. More than $95 \%$ of rejection episodes were biopsy proved and routinely treated with a steroid bolus (1,000 mg. intravenous methylprednisolone) and short recycle with augmentation of the tacrolimus dosage. For steroid resistant rejection $5 \mathrm{mg}$. OKT3 was given intravenously daily for 10 to 14 days. Patients randomized to double therapy were crossed over to receive mycophenolate mofetil if moderate acute or steroid resistant rejection developed. Sporadically, $500 \mathrm{mg}$ / $\mathrm{kg}$. immunoglobulin $\times 4$ was administered intravenously for refractory rejection, ${ }^{17,18}$ that is in patients who had persistent rejection despite steroids, OKT3 and crossover to mycophenolate mofetil.

Randomization and statistical analysis. Continuous variables are presented as mean plus or minus standard deviation and categorical variables as proportions. Randomization was done by sequential draw of assignment using a variable block randomization scheme. ${ }^{19}$ The block sizes varied (4 or 6$)$ and were selected with equal probability. The order of assignment within a block was determined by generating a random number between 0 and 1 , and then rearranging the random numbers in ascending order. Baseline characteristics of the patient population were compared using the standard 2-sample $t$ test for continuous data and Pearson's chi-square test for categorical data.

Patient survival was calculated from the date of kidney transplantation until death and graft survival from the date of kidney transplantation until graft failure, re-transplantation or patient death. Survival curves were generated using the Kaplan-Meier (product limit) method ${ }^{20}$ and compared by the log rank (Mantel-Cox) test. ${ }^{21}$ All analyses were performed according to intention to treat using computer software. All tests were 2 -tailed and $\mathrm{p}<0.05$ was considered statistically significant. Although no investigational agents were used, approval by the Health Sciences Institutional Review Board of the University of Pittsburgh was obtained with yearly renewal because of the randomized nature of the trial.

\section{RESULTS}

Median followup was 8.6 months, which was too short to allow for calculation of 1-year actuarial patient and graft survival statistics. The 6-month actuarial patient survival was $95 \%, 92 \%$ in the double therapy group and $98 \%$ in the triple therapy group (not significant). The 6-month actuarial graft survival was $88 \%, 84 \%$ in the double therapy group and $92 \%$ in the triple therapy group (not significant). The overall incidence of rejection and steroid resistant rejection was 34.2 and $4.2 \%$, respectively (table 2 ). There was a strong trend

TABLE 1. Recipient and donor characteristics

\begin{tabular}{|c|c|c|c|}
\hline & Tacrolimus - Prednisone & Tacrolimus + Prednisone - Mycophenolate Mofetil & Overall \\
\hline No. pts. & 59 & 61 & 120 \\
\hline Mean recipient age $\pm S D$ (range) & $54.0=13.1(19.3-84.1)$ & $47.7 \pm 14.5(18.8-71.7), p<0.02$ & $50.8 \pm 14.1(18.8-84.1)$ \\
\hline No. second transplantation $(T)$ & $6 \quad(10.2)$ & $11 \quad(18.0)$ & $17 \quad(14.2)$ \\
\hline $\begin{array}{l}\text { No. panel reactive antibodies } \\
\text { greater than } 40 \%(\%)\end{array}$ & (3.4) & (3.3) & (3.3) \\
\hline No. previous liver or heart $(\%)$ & (8.5) & $(9.8)$ & (9.2) \\
\hline $\begin{array}{l}\text { No. recipient age greater than } 60 \\
\text { yrs. }\left(C_{(}\right)\end{array}$ & $(37.3)$ & $(26.2)$ & (31.7) \\
\hline Mean donor age $=S D($ range $)$ & $33.8=20.9 \quad(0.3-70.0)$ & $34.7 \pm 22.6(0.01-76.0)$ & $34.3 \pm 21.7(0.01-76.0)$ \\
\hline $\begin{array}{l}\text { No. en bloc donor } 3 \text { years or } \\
\text { younger }(q)\end{array}$ & $10 \quad(16.9)$ & $6 \quad(9.8)$ & $16 \quad(13.3)$ \\
\hline No. donor older than 60 yrs. $(\%)$ & $(13.6)$ & $(16.4)$ & $(15.0)$ \\
\hline Mean ischemia time \pm SD (hrs.) & $29.5=8.5$ & $32.3 \pm 8.2$ & $30.9 \pm 8.4$ \\
\hline $\begin{array}{l}\text { Mean human leukocyte antigen } \\
\text { match } \pm S D\end{array}$ & $2.6=1.3$ & $2.6 \pm 1.2$ & $2.6 \pm 1.2$ \\
\hline $\begin{array}{l}\text { Mean human leukocyte antigen } \\
\text { mismatch }=\mathrm{SD}\end{array}$ & $3.1=1.4$ & $3.1 \pm 1.3$ & $3.1 \pm 1.3$ \\
\hline No. $\mathrm{O}$ antigen mismatch $(\%)$ & (5.1) & (6.6) & 15.81 \\
\hline
\end{tabular}


TABLE 2. Results

\begin{tabular}{|c|c|c|c|}
\hline & \% Tacrolimus + Prednisone & \% Tacrolimus + Prednisone + Mycophenolate Mofetil & \% Overall \\
\hline Rejection & 42.4 & 26.2 & 34.2 \\
\hline Steroid resistant rejection & 6.8 & 1.6 & 4.2 \\
\hline Delayed graft function & 22 & 28 & 25 \\
\hline Cytomegalovirus & 5.1 & $19.7(p<0.03)$ & 12.5 \\
\hline Post-transplantation diabetes mellitus & 10.9 & 8.3 & 9.6 \\
\hline Crossover & $18.6(2 \rightarrow 3)$ & $(3 \rightarrow 2)(p<0.005)$ & 30.8 \\
\hline
\end{tabular}

toward less rejection in the mycophenolate mofetil group than in the double therapy group (26.2 versus $42.4 \%$ ). There was also a trend toward less steroid resistant rejection in the mycophenolate mofetil group ( 1.6 versus $6.8 \%$ ). In the patients who never discontinued mycophenolate mofetil the incidences of rejection and steroid resistant rejection were 14 and $0 \%$, respectively (table 3 ).

The incidence of asymptomatic or symptomatic cytomegalovirus was $12.5 \%$, and was higher in the mycophenolate mofetil group than in the double therapy group (19.7 versus $5.1 \%$, p $<0.03$, table 2). Cytomegalovirus was treated with intravenous ganciclovir and reduction of immunosuppression until the antigenemia level decreased to zero. There were no serious cytomegalovirus related complications. Prophylatic high dose oral acyclovir was given to seropositive recipients. Seronegative recipients of seropositive organs received either ganciclovir and cytomegalovirus hyperimmune globulin or no prophylaxis with frequent antigenemia screening as part of a separate randomized trial.

Post-transplantation lymphoproliferative disorder occurred in a 57-year-old sensitized woman who was randomized to double therapy but received OKT3 and mycophenolate mofetil on postoperative day 1 because of suspected antibody mediated rejection. She eventually achieved normal renal function but multiple nodules of pulmonary lymphoproliferative disorder developed 9 months after transplantation. She was treated with cessation of tacrolimus and mycophenolate mofetil, intravenous ganciclovir and lymphokine activated killer cell therapy, ${ }^{22}$ with regression of the lesions and initially stable renal function. The patient returned to dialysis 3 months later.

There was no significant difference between the 2 groups in delayed graft function $(25 \%$, table 2$)$ or initial posttransplantation diabetes $(9.6 \%)$, which was lower than that reported in earlier trials. ${ }^{1,6,7}$ The routine management of this latter complication has involved gradual reduction of the tacrolimus and prednisone dosages. Historically $50 \%$ of our patients have been able to discontinue insulin after the decrease in dosage. ${ }^{1}$ Followup was too short to determine the final incidence of post-transplantation diabetes in this series.

Crossover between the 2 groups was common. In $42.6 \%$ of patients randomized to triple therapy mycophenolate mofetil was discontinued at 1 time or another (table 2). The most common reason was gastrointestinal toxicity, principally diarrhea, and occasionally gastritis, which was believed to be related to mycophenolate mofetil because it was generally not seen in patients randomized to double therapy. Hematological toxicity, principally neutropenia or thrombocytopenia, was another cause of crossover. Viral infection with cytomegalovirus, Epstein-Barr virus or polyoma $(B K)$ virus was the

TABLE 3. Results of rejection and steroid resistant rejection in the triple therapy group

\begin{tabular}{lccc}
\hline & $\begin{array}{c}\text { Discontinued } \\
\text { Mycophenolate } \\
\text { Mofetil }\end{array}$ & $\begin{array}{c}\text { Never Discontinued } \\
\text { Mycophenolate } \\
\text { Mofetil }\end{array}$ & Overall \\
\hline No. pts. & 26 & 35 & 61 \\
\% Rejection & 42.3 & 14 & 26.2 \\
\% Steroid resis- & 3.8 & 0 & 1.6 \\
tant rejection & & & \\
\hline
\end{tabular}

other main indication for crossover. Even in the absence of crossover reduction of the mycophenolate mofetil dose to 500 mg. orally twice a day was common, mainly for gastrointestinal toxicity. The incidence of crossover declined from the first to the second 6 months of the trial from 48.5 to $35.7 \%$. Mycophenolate mofetil was added because of rejection in $18.6 \%$ of patients randomized to double therapy.

\section{DISCUSSION}

We have used tacrolimus as a primary immunosuppressive agent in our patients undergoing renal transplantation since 1989, and have been impressed with the overall immunosuppressive efficacy, improved half-life, ability to allow steroid withdrawal in the majority of patients with successful transplants, lower cholesterol levels and lower incidence of hypertension. ${ }^{1-12}$ However, the incidence of early acute rejection in our patients, who have not as a rule received induction antilymphocyte therapy, has been relatively high. ${ }^{8-12}$ The consequences of steroid resistant rejection have been frankly disappointing. ${ }^{2,3}$ Thus, we have considered possible third agents with the goal of reducing the rate of rejection, and possibly improving overall patient and graft survival. Although azathioprine was associated with somewhat less rejection, the overall poorer outcomes associated with its use rendered it a poor candidate for routine use as a third agent. ${ }^{2,3}$ Antilymphocyte induction has not been used routinely in our program because of concerns about over immunosuppression. In view of the favorable outcomes achieved with mycophenolate mofetil and cyclosporine based regimens, ${ }^{13-16}$ we thought that it would be worthwhile to evaluate mycophenolate mofetil with tacrolimus.

The preliminary findings suggest that a lower incidence of rejection is achievable with mycophenolate mofetil, and with a greater accrual of patients we would expect to see a statistically significant reduction in rejection. The gastrointestinal and hematological complications seem not to be excessively difficult to manage. Separating the timing of the 2 agents and reducing the dosage of mycophenolate mofetil have usually been successful, and the rate of crossover from triple to double therapy in the second 6 months of the trial was less than in the first 6 months. The higher incidence of cytomegalovirus is of some concern but the explanation may be that the $5.1 \%$ incidence of cytomegalovirus in the double therapy group is perhaps unusually low, rather than the incidence in the triple therapy group being particularly high. Of note, our overall preliminary findings are similar to those of Roth et al, who have reported their retrospective, nonrandomized experience with tacrolimus and mycophenolate mofetil. ${ }^{23}$ They have reported even less rejection in patients receiving myconphenolate mofetil ( 8 versus $21 \%$ of patients not receiving myconphenolate mofetil) presumably because of the routine use of antibody induction.

In conclusion, we are encouraged by the combination of tacrolimus and mycophenolate mofetil, and expect that the associated toxicities will be manageable, in return for a substantial reduction in the incidence of acute rejection. Longer followup will be necessary to determine whether there will be an improvement in patient or graft survival.

Regina Fenton, Loraine Oczypok, Deborah Good, Holly Woods, Jareen Flohr, Sue Bauder, Jennifer Ovesney, Sharon Orlofske, Mark Paynter, and Gerri James assisted with pa- 
tient care, Janet Schmelzer with data entry and organization, Kate Carr with slide preparation and Susan Shandor with tables and slide preparation.

\section{REFERENCES}

1. Shapiro, R., Jordan, M. L., Scantlebury, V. P.. Vivas, C., Fung, J. J., McCauley, J., Randhawa, P., Demetris. A. J., Irish, W., Jain, A., Mitchell, S., Hakala, T. R., Simmons. R. L. and Starzl, T. E.: A prospective, randomized trial of FK506/prednisone vs. FK506/azathioprine/prednisone in renal transplant patients. Transplant. Proc., 27: 814, 1995.

2. Shapiro, R., Jordan, M. L., Scantlebury, V. P., Vivas, C., Gritsch, H. A., Corry, R., Egidi, F., McCauley, J., Demetris, D., Gilboa, N., Lombardozzi-Lane, S., Rao, A., Fontes, P., Zeevi, A., Trucco, M., Demetris, A. J., Randhawa. P.. Irish, W., Fung, J. J., Hakala, T. R., Simmons, R. L. and Starzl, T. E.: The superiority of tacrolimus in renal transplant recipients-the Pittsburgh experience. In: Clinical Transplant 1995. Edited by P. I. Terasaki and J. M. Cecka. Los Angeles: UCLA Tissue Typing Laboratory, pp. 199-205, 1996.

3. Shapiro, R., Jordan, M. L., Scantlebury, V. P., Vivas, C., Gritsch, H. A., McCauley, J., Ellis, D., Gilboa, N., Lombardozzi-Lane, S., Randhawa, P., Demetris, A. J., Irish, W., Hakala, T. R., Simmons, R. L., Fung, J. J. and Starzl, T. E.: Tacrolimus in renal transplantation. Transplant. Proc., 28: 2117, 1996.

4. Shapiro, R., Scantlebury, V. P., Jordan, M. L., Vivas, C., Gritsch, H. A., Ellis, D., Gilboa, N., Lombardozzi-Lane, S., Irish, W., Fung, J. J., Hakala, T. R., Simmons, R. L. and Starzl, T. E. Tacrolimus in pediatric renal transplantation. Transplantation, 62: 1752, 1996.

5. Gjertson, D. W., Cecka, J. M. and Terasaki, P. I.: The relative effects of FK506 and cyclosporine on short- and long-term kidney graft survival. Transplantation, 60: 1384, 1995.

6. Pirsch, J.: FK506 in kidney transplantation: results of the U.S Randomized, comparative, phase III study. FK506 United States Kidney Transplant Multicenter Study Group, Madison Wisconsin. Presented at the ASTP/ASTS Joint Symposium, 1996.

7. Miller, J., Pirsch, J. D., Deierhoi, M., Vincenti, F., Filo, R. S. and the FK 506 Kidney Transplant Study Group: FK 506 in kidney transplantation: results of the U.S.A. randomized comparative phase III study. Transplant. Proc., 29: 304, 1997.

8. Starzl, T. E., Fung, J. J., Jordan, M., Shapiro, R., Tzakis, A., McCauley, J., Johnston, J., Iwaki, Y., Jain, A., Alessiani, M. and Todo, S.: Kidney transplantation under FK506. J.A.M.A., 264: 63, 1990.

9. Shapiro, R., Jordan, M., Fung, J., McCauley, J., Johnston, J., Iwaki, Y., Tzakis, A., Hakala, T., Todo, S. and Starzl, T. E. Kidney transplantation under FK506 immunosuppression. Transplant. Proc., 23: 920, 1991.

10. Shapiro, R., Jordan, M., Scantlebury, V., Fung, J., Jensen, C., Tzakis, A., McCauley, J., Carroll, P., Ricordi, C. and Demetris, A. J.: FK506 in clinical kidney transplantation. Transplant. Proc., 23: 3065, 1991.

11. Shapiro, R., Jordan, M. L., Scantlebury, V. P., Fung, J. J., Jensen, C., Vivas, C., McCauley, J., Irish, W. D., Mitchell, S. and Demetris, A. J.: Randomized trial of FK506/Prednisone vs. FK506/azathioprine/prednisone after renal transplantation: preliminary report. Transplant. Proc.. 25: 669, 1993.

12. Shapiro, R., Scantlebury, V., Jordan, M. L., Vivas, C., Tzakis, A. G., Ellis, D., Gilboa, N., Hopp, L., McCauley, J. and Irish W.: FK506 in pediatric kidney transplantation-primary and rescue experience. Ped. Nephrol., 9: S $43,1995$.

13. Placebo-controlled study of mycophenolate mofetil combined with cyclosporin and corticosteroids for prevention of acute rejection. European Mycophenolate Mofetil Cooperative Study Group. Lancet, 345: 1321, 1995.

14. Sollinger. H. W.: Mycophenolate mofetil for the prevention of acute rejection in primary cadaveric renal allograft recipients. U.S. Renal Transplant Mycophenolate Mofetil Study Group. Transplantation, 60: 225, 1995.

15. A blinded, randomized clinical trial of mycophenolate mofetil for the prevention of acute rejection in cadaveric renal transplantation. Tricontinental Mycophenolate Mofetil Renal Transplant Study Group. Transplantation. 61: 1029, 1996.

16. Halloran, P., Mathew, T., Tomlanovich, S., Groth. C., Hooftman, L. and Barker, C.: Mycophenolate mofetil in renal allograft recipients: a pooled efficacy analysis of three randomized, double-blind, clinical studies in prevention of rejection. The International Mycophenolate Mofetil Renal Transplant Study Groups. Transplantation, 63: 39, 1997.

17. Toyoda, M., Zhand, X. M., Petrosian, A., Wachs, K., Moudgil, A. and Jordan, S. C.: Inhibition of allospecific responses in the mixed lymphocyte reaction by pooled human gamma-globulin. Transplant. Immunol., 2: 337, 1994.

18. Czer, L., Jordan, S. C. and Tyan, D.: Novel approach to treatment of patients with cytotoxic antibodies after heart transplantation: use of IVIG. Circulation, 88: 494, 1989.

19. Friedman, L. M., Furberg, C. D. and DeMets, D. L.: Fundamentals of Clinical Trials, 2nd ed. St. Louis: C. V. Mosby Co., p. 51, 1985.

20. Kaplan, E. and Meier, P.: Nonparametric estimation from incomplete observations. J. Amer. Stat. Assn., 53: 457, 1958.

21. Mantel, N.: Evaluation of survival data and two new rank order statistics arising in its consideration. Cancer Chemother. Rep., 50: $163,1966$.

22. Nalesnik, M., Rao, A., Elder, E., Rowe, D., Rybka, W., Zeevi, A., Whiteside, T. W., Pham, S., Keenan, R., Griffith, B., Fung, J. J. and Starzl, T. E.: Treatment of posttransplant lymphoproliferative disease (PTLD) in solid organ recipients by infusion of autologous lymphokine-activated peripheral blood leukocytes (PBL). Presented at the XVI International Congress of the Transplantation Society, Barcelona, 1996.

23. Roth, D., Colona, J., Burke, G., Cianco, G., Esquenazi, J. and Miller, J.: Primary immunosuppression with FK506 and mycophenolate mofetil for renal allograft recipients. Presented at the 16th Annual Meeting of the American Society of Transplant Physicians, Chicago, 1997.

\section{EDITORIAL COMMENT}

The estimated half-life of renal allografts is shorter for recipients who have acute rejection episodes compared to those who do not. Mycophenolate mofetil is associated with a 60 to $70 \%$ decrease in acute rejection episodes. ${ }^{1.2}$ Thus, it is hopeful that this immunosuppressive drug will also increase long-term half-life allograft survival in kidney recipients. This hope is driven by encouraging results from several large studies (reference 15 of article). ${ }^{2}$ The authors present a randomized, prospective trial of 120 patients comparing 2 tacrolimus based regimens with $(61)$ and without $(59) 2 \mathrm{gm}$. mycophenolate mofetil daily. The groups were similar in all characteristics except for recipient age as the mycophenolate mofetil group was younger than the double therapy group $(47.7$ versus 54 years, $p<0.02)$. Nevertheless, other characteristics seemed different but were not statistically significant (second transplantation 10.2 versus $18 \%$, recipients older than 60 years 37.3 versus $26.2 \%$, donors younger than 3 years old with en bloc kidneys 16.9 versus $9.8 \%$ and cold ischemia hours 29.5 versus 32.3 , respectively, with and without mycophenolate mofetil). Medium followup was short $(8.6 \pm 0.5$ months) and the data were reported in terms of 6-month actuarial graft survival (84 versus $92 \%$ with and without mycophenolate mofetil). Rejection rates were 42.4 versus $26.2 \%$ and steroid resistant 6.8 versus $1.6 \%$, respectively, with and without mycophenolate mofetil. None of these comparisons approached statistical significance. However, there were significant increases in complications reported in the mycophenolate mofetil group (19.7 versus $5.1 \%$, p $<0.03$ ) for cytomegalovirus and 42.6 versus $18.6 \%$ ( $p<0.005$ ) crossing over predominantly because of gastrointestinal toxicity.

While this study was randomized and prospective, it was not blinded. There are many factors that can significantly dilute the results so that significance is not seen, as in many other studies comparing cyclosporine double therapy to that with the same regimen containing mycophenolate mofetil. These factors may be the inclusions of patients with heart/kidney and liver/kidney transplants who are different immunologically from those who have received kidney transplants only. Another factor that can confuse the results is the crossover from the mycophenolate mofetil group mainly because gastrointestinal toxicity. Gastrointestinal toxicity was noted to be much more serious than in other nontacrolimus, that is cyclosporine A, studies, which may reflect on a potential synergistic effect that mycophenolate mofetil and tacrolimus have on the gastrointestinal tract. The European Mycophenolate Mofetil Cooperative Study Group reported only a $17.6 \%$ withdrawal rate due to adverse events in 165 patients on similar $2 \mathrm{gm}$. mycophenolate mofetil daily.2 Although it is stated that these symptoms can be "relatively straight 
forward to manage," a significant consequence of gastrointestinal toxicity is inadequate absorption of medications and, thus, suboptimal immunosuppression, perhaps contributing to greater rejection risk. The authors noted that the trend towards less rejection was not significant. Unfortunately, these preliminary findings may not have the statistical power to offer definitive conclusions. Finally, the relatively low graft survival noted in a short followup (gold standard for followup is 1-year graft survival) is of major concern, since graft loss at 1 year should be predictably greater.

Nevertheless, this study offers a large experience with the use of tacrolimus and mycophenolate mofetil although the data are difficult to assess and it is too soon to derive assumptions. It is apparent that mycophenolate mofetil and tacrolimus compared to mycophenolate mofetil and cyclosporine ${ }^{2}$ are associated with greater gastrointestinal toxicity and cytomegalovirus infections. Whether this difference justifies the possibility of better acute rejection prevention remains to be seen. I agree with the authors that longer followup is necessary.

Peter N. Bretan, Jr.

Renal Transplant Service

University of California, San Francisco Medical Center San Francisco, California

1. Tesi, R. J., Henry, M. L., Elkammas, E. A. and Ferguson, R. M.: Predictors of long-term primary cadaveric renal transplant survival. Clin. Transplant., 7: 345, 1993.

2. Wiesel, M. and Carl, S. for the European Mycophenolate Mofetil Cooperative Study Group. A placebo controlled study of mycophenolate mofetil used in combination with cyclosporine and corticosteroids for the prevention of acute rejection in renal allograft recipients: 1-year results. J. Urol., 159: 28, 1998. 